\title{
A Multiphysics Strategy for Free Surface Flows
}

\author{
Edie Miglio, Simona Perotto, and Fausto Saleri \\ MOX, Modeling and Scientific Computing, Department of Mathematics, \\ Politecnico of Milano, via Bonardi 9, I-20133 Milano, Italy \\ (http://mox.polimi.it/)
}

\begin{abstract}
Summary. This work is the first step towards a multiphysics strategy for freesurface flows simulation. In particular, we present a strategy to couple one and two-dimensional hydrostatic free surface flow models. We aim to reduce the computational cost required by a full $2 \mathrm{D}$ model. After introducing the two models along with suitable a priori error estimates, we discuss the choice of convenient matching conditions stemming from the results obtained in Formaggia et al. [2001]. The numerical results in the last section confirm the soundness of our analysis.
\end{abstract}

\section{Introduction}

Final aim of our research is an efficient and accurate numerical simulation of the motion of water in a complex system of channels such as, for instance, the well-known Venice lagoon. A hydrodynamic configuration of this type involves a wide spectrum of space and time scales related to the presence of different physical phenomena. It is well known that in hydrodynamics there exists a hierarchy of models derived from the Navier-Stokes equations for an incompressible free-surface fluid. Essentially we can distinguish among 1D, $2 \mathrm{D}$ and $3 \mathrm{D}$ models of hydrostatic and non-hydrostatic type. In descending order of complexity, for the 3D case we can consider either the free surface Navier-Stokes or the hydrostatic 3D shallow water equations; concerning the 2D situation the Boussinesq, Serre or Saint-Venant equations can be adopted; finally the $1 \mathrm{D}$ counterpart of these latter models can be used (see, e.g., Miglio et al. [1999], Vreugdenhil [1998], Whitham [1974]). In particular, in this paper we consider only shallow water models, suitable for configurations where the vertical scales are much smaller than the corresponding horizontal ones.

Ideally one should use a full 3D model to capture all the physical features of the problem at hand. However, this approach is characterized by a huge computational effort. Thus, the basic idea is to reduce the computational cost by solving the more expensive model only in some parts of the domain. In this work we deal with the coupling of the $2 \mathrm{D}$ and $1 \mathrm{D}$ shallow water models. 
This choice turns out to be reasonable for instance in the presence of a river bifurcation such as the one shown in Fig. 1 (right). We extend the analysis provided in Formaggia et al. [2001], where the 3D Navier-Stokes equations are coupled with a convenient $1 \mathrm{D}$ model for the description of blood flow in a compliant vessel, to the case of free surface flows. Even if, in our case, the dimension of the coupled models is different, we resort to a similar analysis to derive the suitable coupling conditions.

The outline of the paper is as follows. Sect. 2 deals with the $2 \mathrm{D}$ model. In Sect. 3, we provide the 1D model and a corresponding stability analysis. In Sect. 4 , a set of interface conditions for sub-critical flows is proposed in order to couple the two models. Finally, numerical results are presented in Sect. 5.

\section{The 2D Model}

We consider the description of the motion of a free-surface viscous incompressible fluid when the vertical scales are much smaller compared with the corresponding horizontal ones. This allows us to consider the shallow water theory whose leading hypothesis is the hydrostatic approximation of the pressure, i.e., the pressure of the fluid is assumed to depend on the total water depth only. Many of the 1D and 2D hydrodynamic models, successfully used in practical applications, depart from this assumption.

The 2D model is represented by the Saint-Venant or shallow water equations whose conservative form reads as follows

$$
\begin{cases}\frac{\partial(h \mathbf{U})}{\partial t}+\nabla \cdot(h \mathbf{U} \otimes \mathbf{U})+g h \nabla h=\mathbf{0} & \text { with } \quad \mathbf{x} \in \Omega \quad \text { and } t>0 \\ \frac{\partial h}{\partial t}+\nabla \cdot(h \mathbf{U})=0 & \text { with } \quad \mathbf{x} \in \Omega \quad \text { and } t>0\end{cases}
$$

where $\mathbf{x}=(x, y)^{T}, \mathbf{U}=(u, v)^{T}$ is the average velocity, $h$ denotes the total water depth and $\Omega \subset \mathbb{R}^{2}$ is a bounded open set. Of course, system (1) has to be provided with suitable initial and boundary conditions (see, e.g., Agoshkov et al. [1993]). We assume to be in the presence of a flat bottom and the effect of the friction is neglected. Moreover, we are interested in sub-critical flow regimes. The theory on hyperbolic systems can be applied to compute the eigenvalues and eigenfunctions of (1). With this aim, by considering a region of smooth flow, we can obtain the quasi-linear form of (1)

$$
\frac{\partial \mathbf{W}}{\partial t}+A(\mathbf{W}) \frac{\partial \mathbf{W}}{\partial x}+B(\mathbf{W}) \frac{\partial \mathbf{W}}{\partial y}=\mathbf{0},
$$

where $\mathbf{W}=(u, v, h)^{T}$,

$$
A=\left[\begin{array}{lll}
u & 0 & g \\
0 & u & 0 \\
h & 0 & u
\end{array}\right] \quad \text { and } B=\left[\begin{array}{ccc}
v & 0 & 0 \\
0 & v & g \\
0 & h & v
\end{array}\right]
$$


It is well known that the eigenvalues of system (1) are

$$
\mu_{1}=-(\cos (\phi) u+\sin (\phi) v), \quad \mu_{2,3}=-(\cos (\phi) u+\sin (\phi) v) \pm \sqrt{g h}
$$

$\phi$ being the direction of the characteristic lines, while the associated eigenfunctions are given by

$$
w_{1}=\left[\begin{array}{c}
\sin (\phi) \\
-\cos (\phi) \\
0
\end{array}\right], \quad w_{2,3}=\left[\begin{array}{c} 
\pm \sqrt{g / h} \cos (\phi) \\
\pm \sqrt{g / h} \sin (\phi) \\
1
\end{array}\right]
$$

Concerning the stability analysis, a priori results are available in the literature for the Saint-Venant equations in the conservative form and provided with suitable boundary conditions (see, for instance, Agoshkov et al. [1993]).

\section{The 1D Model}

In the case $\Omega$ is an open channel, the $2 \mathrm{D}$ Saint-Venant equations (1) can be replaced by a $1 \mathrm{D}$ shallow water model, by assuming that the velocity is uniform over any cross section, that the channel is sufficiently straight and its slope sufficiently mild and uniform throughout the region. Moreover, the streamwise bottom slope and the lateral inflow are assumed equal to zero and the bottom friction is neglected as in the $2 \mathrm{D}$ model.

We focus on the case of one-dimensional channels with a rectangular crosssection. This choice turns out not to be so restrictive in realistic situations. Indeed, even if the cross-section is irregular, a sophisticated channel schematization can be employed by resorting to rectangular sections (see Schulz and Steinebach [2002]). In such a case the 1D model reduces to the system

$$
\begin{cases}\frac{\partial A}{\partial t}+\frac{\partial Q}{\partial x}=0 & \text { with } x \in(a, b) \text { and } t>0 \\ \frac{\partial Q}{\partial t}+\frac{\partial}{\partial x}\left(\frac{Q^{2}}{A}\right)+g A \frac{\partial h}{\partial x}=0 & \text { with } x \in(a, b) \text { and } t>0\end{cases}
$$

where $A$ is the area of the wet cross-section, $Q$ is the discharge and $h$ denotes the total water depth. System (2) has to be supplied with proper boundary conditions. Without reducing the generality of our analysis, we assume that the algebraic relation

$$
h=\psi(A)+h_{0}, \quad \text { with } \quad \frac{\partial \psi}{\partial A}>0 \quad \text { and } \quad \psi\left(A_{0}\right)=0
$$

holds between the total water depth and the area. Here $h_{0}$ stands for the constant undisturbed water depth, $A_{0}$ is the area of the corresponding wet section while $\psi(A)=\left(A-A_{0}\right) / L, L$ being the width of the section. 
Assumption (3) allows us to rewrite (2) as

$$
\begin{cases}\frac{\partial A}{\partial t}+\frac{\partial Q}{\partial x}=0 & \text { with } x \in(a, b) \quad \text { and } t>0 \\ \frac{\partial Q}{\partial t}+2 \frac{Q}{A} \frac{\partial Q}{\partial x}+\left(g A \frac{\partial h}{\partial A}-\frac{Q^{2}}{A^{2}}\right) \frac{\partial A}{\partial x}=0 & \text { with } x \in(a, b) \quad \text { and } t>0\end{cases}
$$

We start from the quasi-linear form (4) to study the mathematical properties of the solutions of the $1 \mathrm{D}$ model. Under the assumption (3), it can be proved that system (2) is hyperbolic since it has two real eigenvalues $\lambda_{1,2}=u \pm c$, where $u=Q / A$ while

$$
c(A)=\sqrt{g A \frac{\partial \psi(A)}{\partial A}}=\sqrt{g h}
$$

is the celerity of the system. In such a case it is also possible to compute the characteristic variables given by

$$
W_{1,2}=u \pm \int_{A_{0}}^{A} \frac{c(\tau)}{\tau} d \tau=u \pm \int_{A_{0}}^{A} \sqrt{\frac{g \tau}{L}} \frac{1}{\tau} d \tau=u \pm 2 \sqrt{\frac{g}{L}}\left[\sqrt{A}-\sqrt{A_{0}}\right]
$$

\subsection{Stability Analysis}

In this section we provide an a priori estimate for system (2).

We assume that, for any time $t>0$, the area $A$ remains positive and that the eigenvalues $\lambda_{1}$ and $\lambda_{2}$ are of opposite $\operatorname{sign}\left(\lambda_{1}>0, \lambda_{2}<0\right)$, that is we consider a sub-critical and unidirectional flux. This is the most interesting situation in view of the coupling with the 2D model. We endow system (2) with the following general initial and boundary conditions:

$$
\begin{gathered}
A(x, 0)=A^{*}(x), \quad Q(x, 0)=Q^{*}(x) \text { with } a<x<b, \\
W_{1}=g_{1}(t) \text { at } x=a, \quad W_{2}=g_{2}(t) \text { at } x=b, \quad \text { with } t>0 .
\end{gathered}
$$

Let us introduce the energy associated with model (2), defined, for any $t>0$, as

$$
E(t)=\frac{1}{2 g} \int_{a}^{b} A(x, t) u^{2}(x, t) d x+\int_{a}^{b} \Psi(A(x, t)) d x
$$

with $\Psi(A)=\int_{A_{0}}^{A} \psi(\tau) d \tau$. Thanks to (3), we can guarantee that $\Psi(A)$ and the energy $E(t)$ are positive functions, for any $t>0$ and for any $Q$ and $A$ strictly positive.

Thus, the following conservation property can be proved.

Lemma 1. Let us assume that relation (3) holds. Then for any $T>0$, we have

$$
E(T)+\left.\int_{0}^{T} Q\left(\left(h-h_{0}\right)+\frac{1}{2 g} u^{2}\right)\right|_{a} ^{b} d t=E(0),
$$

$E(0)$ depending only on the initial values $A^{*}$ and $Q^{*}$. 
We refer to Miglio et al. [2003] for the proof of this result as well as for inequality (7). Result (6) can be used to derive an energy estimate for the 1D problem (2).

Proposition 1. Let us assume that the boundary data $g_{1}$ and $g_{2}$ satisfy the following restrictions

$$
g_{1}(t)>-2 \sqrt{\frac{g A_{0}}{L}} \quad \text { and } \quad g_{2}(t)<2 \sqrt{\frac{g A_{0}}{L}} .
$$

Then, there exists a positive function $F=F\left(g_{1}, g_{2}, \frac{A_{0}}{L}\right)$ such that

$$
E(T) \leq E(0)+\int_{0}^{T} F\left(g_{1}(t), g_{2}(t), \frac{A_{0}}{L}\right) d t
$$

i.e., the 1D model problem (2) provided with conditions (5) is stable.

Remark 1. If homogeneous boundary conditions are chosen in (5), estimate (7) simplifies to $E(T) \leq E(0)$, provided that $2 \sqrt{A_{0}} / 3<\sqrt{A}<2 \sqrt{A_{0}}$.

We remark that no energy estimate is available for a general cross-section.

\section{Coupling of the Two Models}

After having proved the well-posedness of both the 1D and the 2D problems, we can analyze the coupling of the two models.

Let us consider the coupling sketched on the left of Fig. 1. We denote with $a$ the matching point of the two models. The cross-section at $x=a$ is assumed to be a rectangle and its outward normal is along the $x$-direction. At the righthand side of $a$, i.e., in $\omega$, we solve the $1 \mathrm{D}$ model (2) which provides the physical quantities $A_{1 \mathrm{D}}, Q_{1 \mathrm{D}}$ and $h_{1 \mathrm{D}}$ (and, as a consequence, $u_{1 \mathrm{D}}=Q_{1 \mathrm{D}} / A_{1 \mathrm{D}}$ ). At the left-hand side of $a$, that is in $\Omega$, the 2D Saint-Venant equations (1) are solved and the associated physical quantities are $A_{2 \mathrm{D}}, Q_{2 \mathrm{D}}$ and $h_{2 \mathrm{D}}$ to be defined shortly.
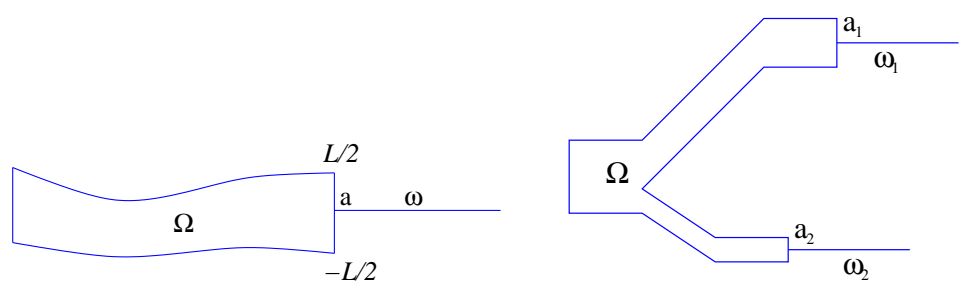

Fig. 1. Coupling of $2 \mathrm{D}$ with $1 \mathrm{D}$ models 
As we are linking quantities of different dimension, we can, for instance, reduce the $2 \mathrm{D}$ ones to one-dimensional information by averaging the two-dimensional terms. With this aim, let us introduce

$$
\bar{u}_{2 \mathrm{D}}=\frac{1}{L} \int_{-\frac{L}{2}}^{\frac{L}{2}} u(a, y) d y, \bar{h}_{2 \mathrm{D}}=\frac{1}{L} \int_{-\frac{L}{2}}^{\frac{L}{2}} h(a, y) d y=\frac{A_{2 \mathrm{D}}}{L}, \bar{Q}_{2 \mathrm{D}}=A_{2 \mathrm{D}} \bar{u}_{2 \mathrm{D}},
$$

i.e., the mean velocity, the mean total water depth and the mean discharge, where $A_{2 \mathrm{D}}=\int_{-\frac{L}{2}}^{\frac{L}{2}} h(a, y) d y$. Due to the unidirectional flow assumption, from the $2 \mathrm{D}$ to the $1 \mathrm{D}$ model, we have a subcritical outflow for the $2 \mathrm{D}$ system (with two outgoing characteristics) and a subcritical inflow for the one-dimensional problem (with an incoming characteristic). It seems reasonable from a physical view-point to demand the continuity of the following quantities at the interface $x=a$ :

- C1. cross-section area: $A_{2 \mathrm{D}}=A_{1 \mathrm{D}}$, with $A_{1 \mathrm{D}}=\bar{h}_{2 \mathrm{D}} L$;

- C2. discharge: $\bar{Q}_{2 \mathrm{D}}=Q_{1 \mathrm{D}}$;

- C3. entering characteristic: $2 \overline{\sqrt{h_{2 \mathrm{D}} g}}+\bar{u}_{2 \mathrm{D}}=2 \sqrt{h_{1 \mathrm{D}} g}+\frac{Q_{1 \mathrm{D}}}{A_{1 \mathrm{D}}}$.

Notice that all the mean variables are considered in an average form on the $2 \mathrm{D}$ problem. On the other hand, concerning the choice of the matching conditions, we remark that $\mathrm{C} 1$. and $\mathrm{C} 3$. would suffice as $\mathrm{C} 2$. is automatically guaranteed when C1. and C3. are satisfied.

\subsection{The Sub-Domain Iteration Algorithm}

To develop a splitting procedure to solve the coupled 1D-2D problem, we enforce at the matching point $x=a$ only those conditions which guarantee the well-posedness of each subproblem in $\Omega$ and $\omega$. With this aim, we exploit the results of the stability analysis above. In particular,

- $\mathrm{C} 1$. is used for imposing the total depth at the outflow of the $2 D$ model;

- C3. is used at the inflow of the $1 D$ model.

Then each subproblem is completed with other boundary conditions:

- condition a: at the inflow of the 2D model we assign the total water depth $h(t)$ as a function of time;

- condition $b$ : at the outflow of the $1 D$ model a non-reflecting boundary condition is employed.

Moreover, we recall that on the rigid walls of the channel, no slip boundary conditions are assigned.

Thus the main steps of the algorithm are: given the solution of the coupled problem at time $t^{n}$, for $k=1,2, \ldots$

1. solve the $2 \mathrm{D}$ problem with $\mathrm{C} 1$. plus condition $a$ in order to obtain $h_{k}^{n+1}$, $U_{k}^{n+1}$; 
2. compute $2 \sqrt{\sqrt{h_{k}^{n+1} g}}+\bar{U}_{k}^{n+1}$, that is an approximation for the left-hand side of $\mathrm{C} 3$;

3. solve the $1 \mathrm{D}$ problem with $\mathrm{C} 3$. plus condition $b$.

We iterate until the coupling conditions are satisfied within a fixed tolerance. In practice, it can be verified that, after 2 or 3 iterations, the difference between the $1 \mathrm{D}$ and averaged $2 \mathrm{D}$ values is very small.

\section{Numerical Assessment}

To test the effectiveness of the proposed algorithm we consider the case of a river bifurcation as sketched on the right of Fig. 1 . We want to solve the $2 \mathrm{D}$ model only in $\Omega$, i.e., near the bifurcation while the one-dimensional problem is solved in $\omega_{1}$ and $\omega_{2}$. The numerical solution of the $2 \mathrm{D}$ model is obtained by using the $2 \mathrm{D}$ counterpart of the approach proposed in Miglio et al. [1999]. As for the 1D model a finite volume method is employed. As initial condition

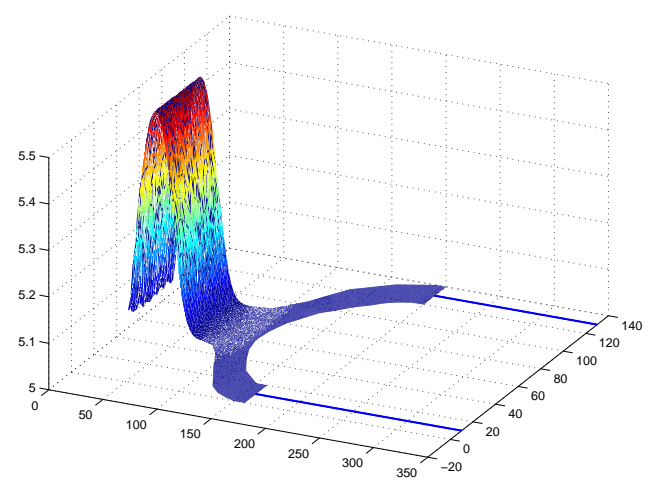

Fig. 2. Initial elevation profile

for the elevation we choose the profile shown in Fig. 2, while the time step is chosen equal to $\Delta t=0.1 \mathrm{~s}$ and for the space discretization of both the $1 \mathrm{D}$ and $2 \mathrm{D}$ models a mesh size $h=0.1 \mathrm{~m}$ is used. In Fig. 3 we show two snapshots of the approximate elevations provided by the full $2 \mathrm{D}$ shallow water model (on the left) and by the coupled 2D-1D one (on the right), respectively, corresponding to two different times $(t=250 \mathrm{~s}$ and $t=300 \mathrm{~s})$. These results confirm the soundness of the algorithm proposed in Sect. 4.1. The wave travels from the $2 \mathrm{D}$ to the $1 \mathrm{D}$ model without any significant distortion: no wave amplitude reduction and no phase difference is evident.

Acknowledgement. This work has been supported by the project MIUR 2001 "Numerical Methods in Fluid Dynamics and Electromagnetism". We thank Prof. Luca Formaggia for useful comments and suggestions. 

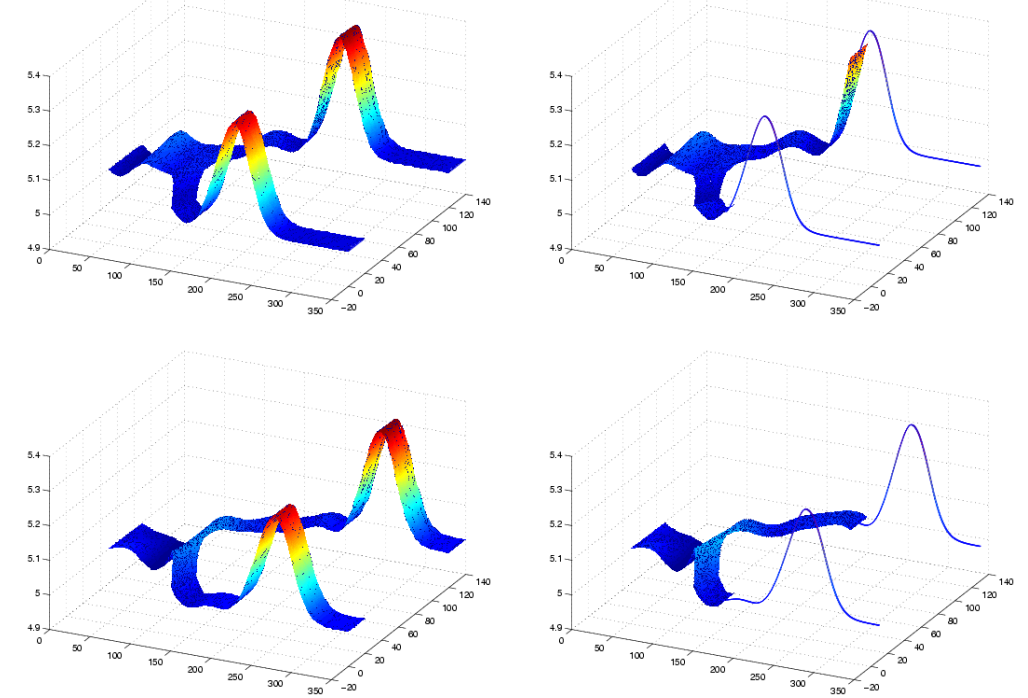

Fig. 3. Approximate elevation for the full $2 \mathrm{D}$ model (on the left) and for the coupled $2 \mathrm{D}-1 \mathrm{D}$ model (on the right) corresponding to $t=250 \mathrm{~s}$ (top) and to $t=300 \mathrm{~s}$ (bottom)

\section{References}

V. Agoshkov, D. Ambrosi, V. Pennati, A. Quarteroni, and F. Saleri. Mathematical and numerical modelling of shallow water flow. Comput. Mech., 11:280-299, 1993.

L. Formaggia, J. Gerbeau, F. Nobile, and A. Quarteroni. On the coupling of 3D and 1D Navier-Stokes equations for flow problems in compliant vessels. Comput. Methods Appl. Mech. Engrg., 191:561-582, 2001.

E. Miglio, S. Perotto, and F. Saleri. On the coupling of 2D and 1D shallow water models for the simulation of free surface flows. in preparation, 2003.

E. Miglio, A. Quarteroni, and F. Saleri. Finite element approximation of quasi-3D shallow water equations. Comput. Methods Appl. Mech. Engrg., 174(3-4):355-369, 1999.

M. Schulz and G. Steinebach. Two-dimensional modelling of the river Rhine. J. Comput. Appl. Math., 145(1):11-20, 2002.

C. Vreugdenhil. Numerical methods for shallow-water flows. Kluwer Academic Press, Dordrecht, 1998.

G. Whitham. Linear and nonlinear waves. Wiley, New York, 1974. 